Supporting information to

\title{
Cylindrical Water Triboelectric Nanogenerator via Controlling Geometrical Shape of Anodized Aluminum for Enhanced Electrostatic Induction
}

Sukyung Lee, ${ }^{\mathrm{a}, \dagger}$ Jihoon Chung, ${ }^{\mathrm{a}, \dagger}$ Dae Yun Kim, ${ }^{\mathrm{a}}$ Jung-Yeul Jung, ${ }^{\mathrm{b}}$ Seong Hyuk Lee ${ }^{\mathrm{a}, *}$ and Sangmin Lee ${ }^{\mathrm{a}, *}$

${ }^{a}$ School of Mechanical Engineering, Chung-Ang University, 84, Heukseuk-ro, Dongjack-gu, Seoul 156-756, Korea

${ }^{\mathrm{b}}$ Technology Center for Offshore Plant Industries, Korea Research Institute of Ships and Ocean Engineering, KIOST, Daejeon 34103, Korea

Prof. Sangmin Lee

*Email: slee98@cau.ac.kr

Homepage: http://slee.cau.ac.kr

Prof. Seong Hyuk Lee

E-mail: shlee89@cau.ac.kr

Homepage: http://cau.ac.kr/ mtel/

${ }^{\dagger}$ These authors contributed equally to this work 

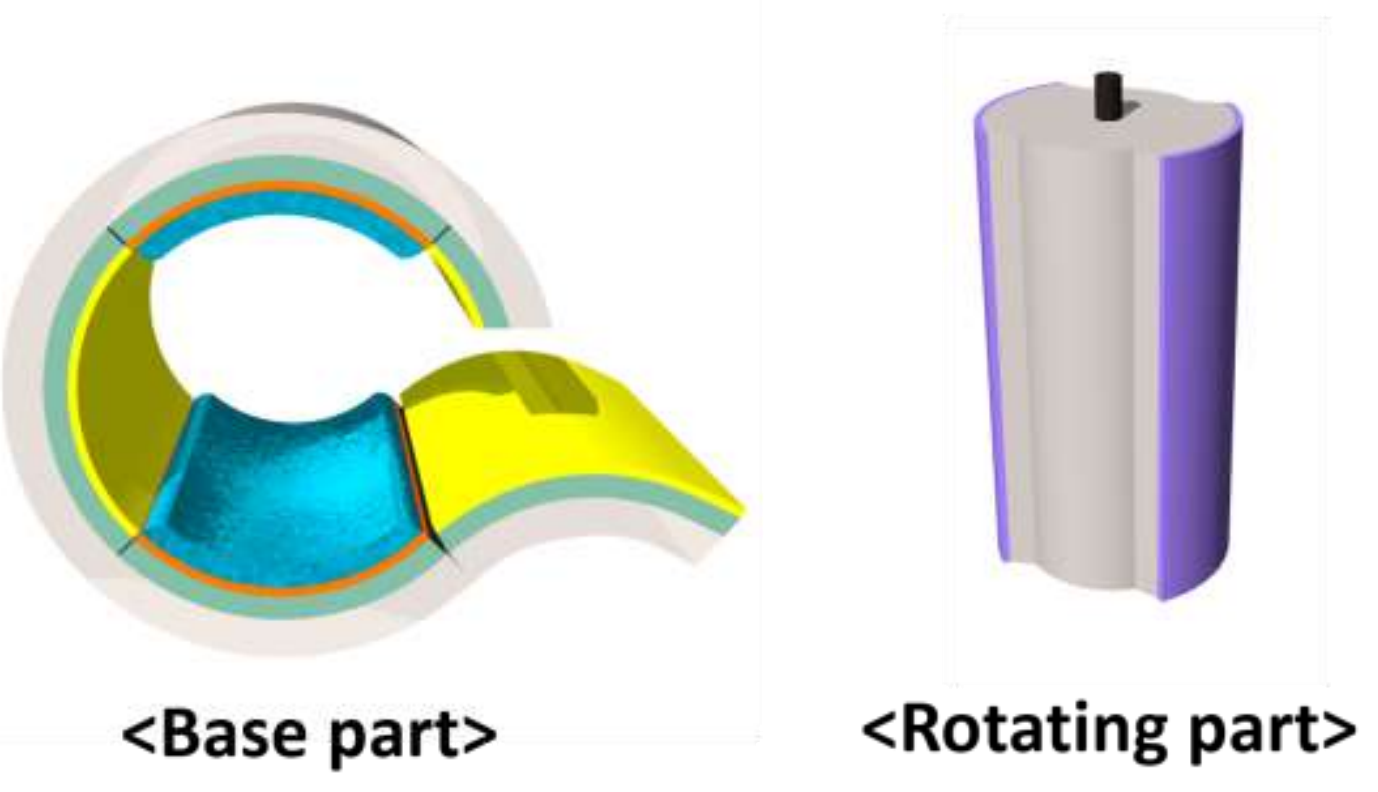

Figure S1. Schematic illustration of base part and rotating part of CW-TENG. 


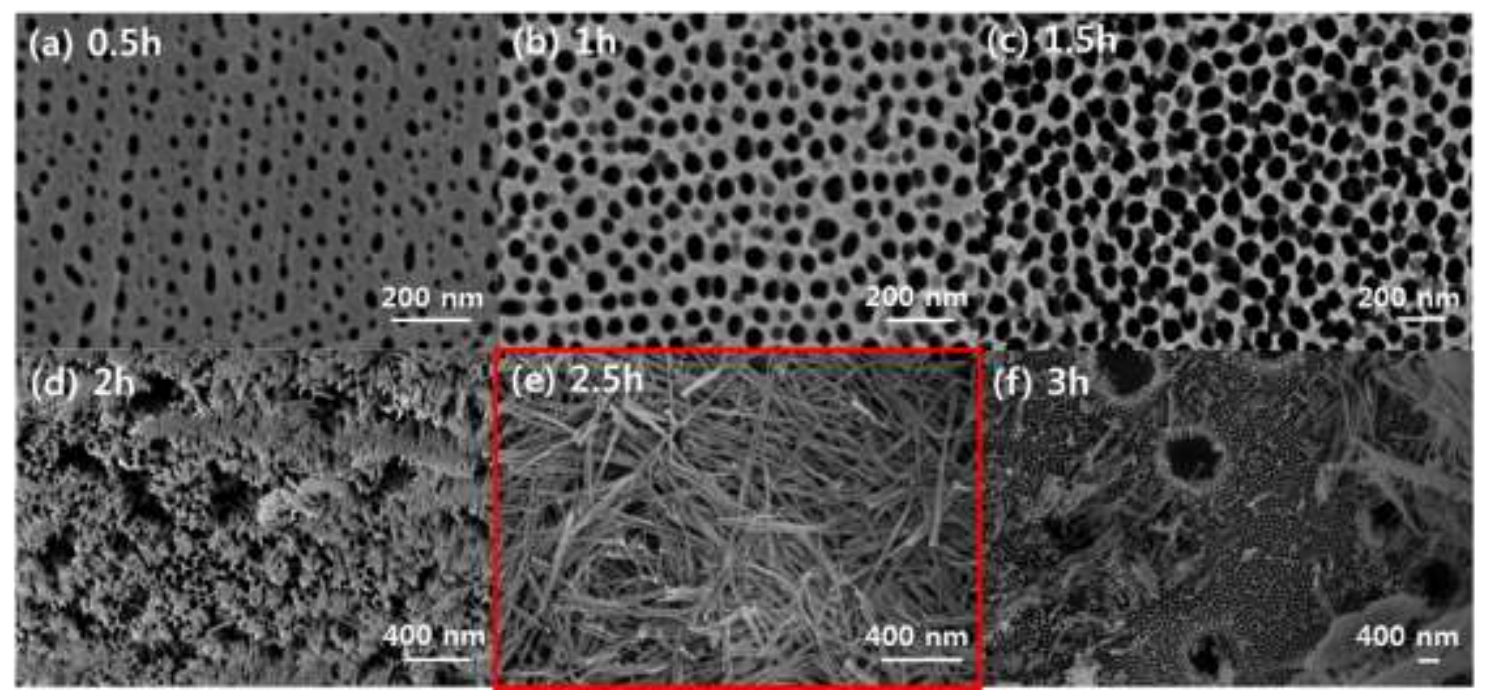

Figure S2. FE-SEM image of AAO structure according to widening time. 

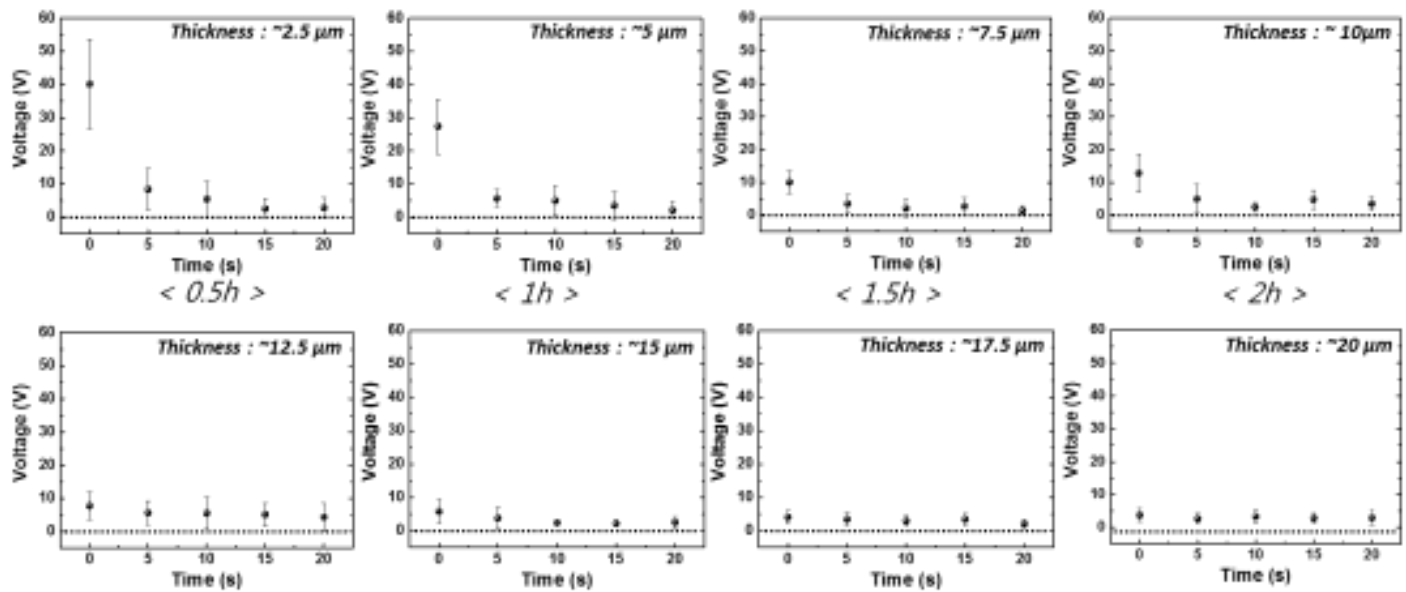

$<2.5 h>$

$<3 h>$

$<3.5 h>$

$<4 h>$

Figure S3. Continuous voltage output of hydrophobic surface according to anodizing time. 


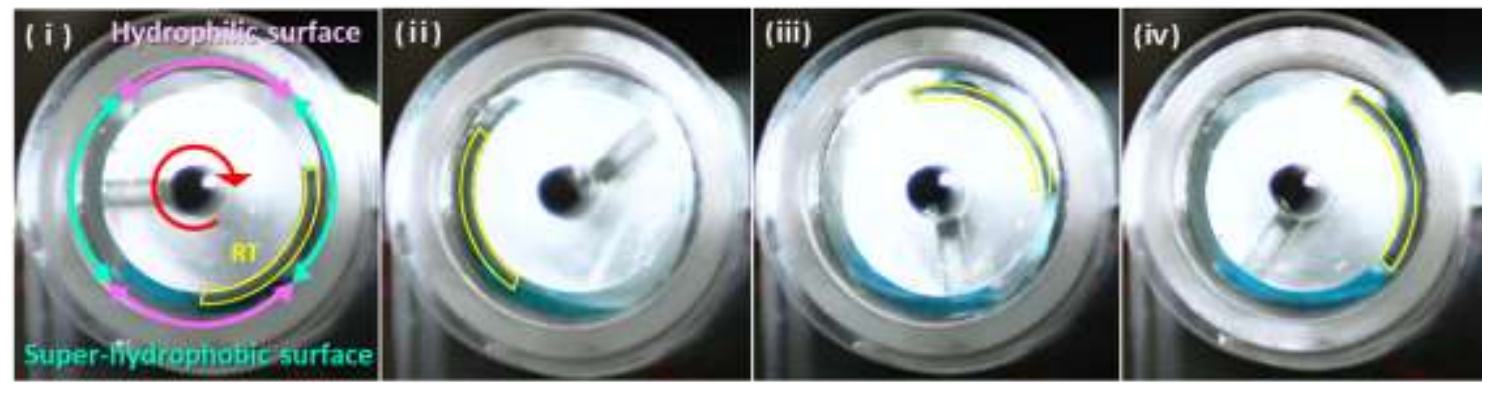

Figure S4. High-speed photographs of water behavior in the CW-TENG 

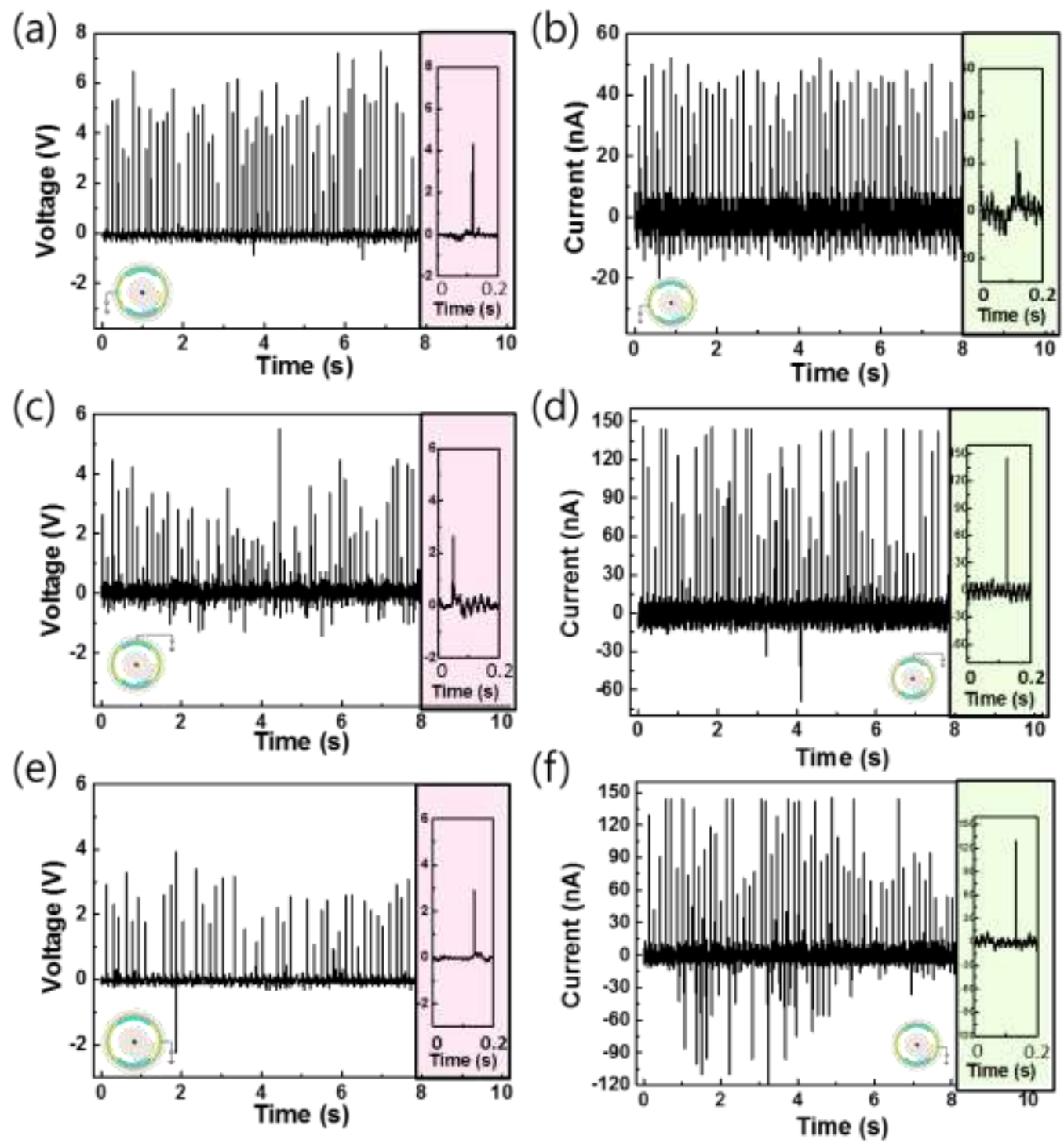

Figure S5. Output performance of super-hydrophobic surface TENG, and other generation units. Open-circuit voltage $\left(V_{O C}\right)$ and Shor-circuit current of (a) left, (b) upper and (c) right generation units. 


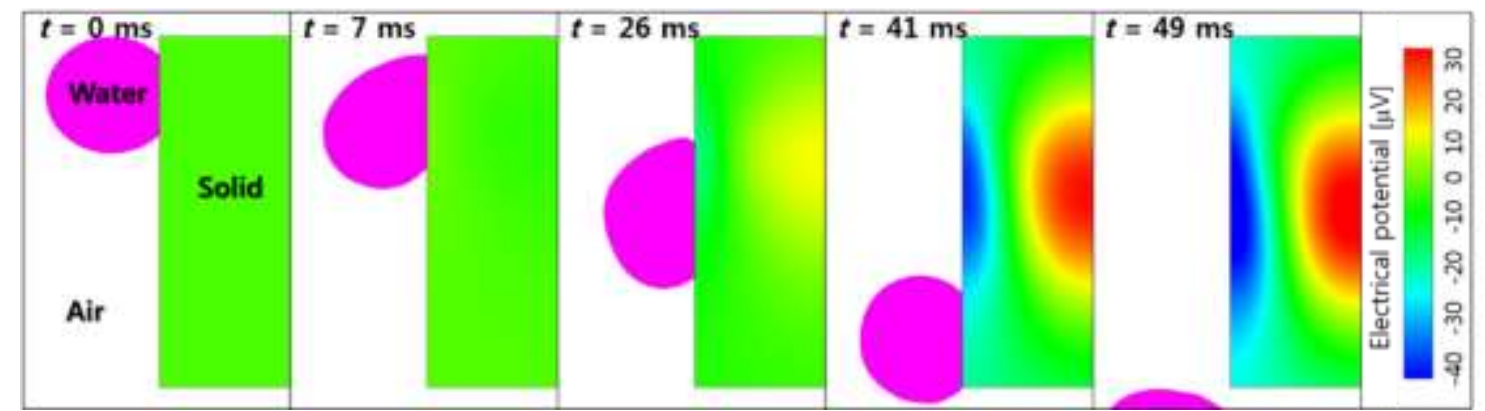

Figure S6. Simulation results of electrical potential field inside the electrode with respect to water drop falling off from the hydrophobic surface. 

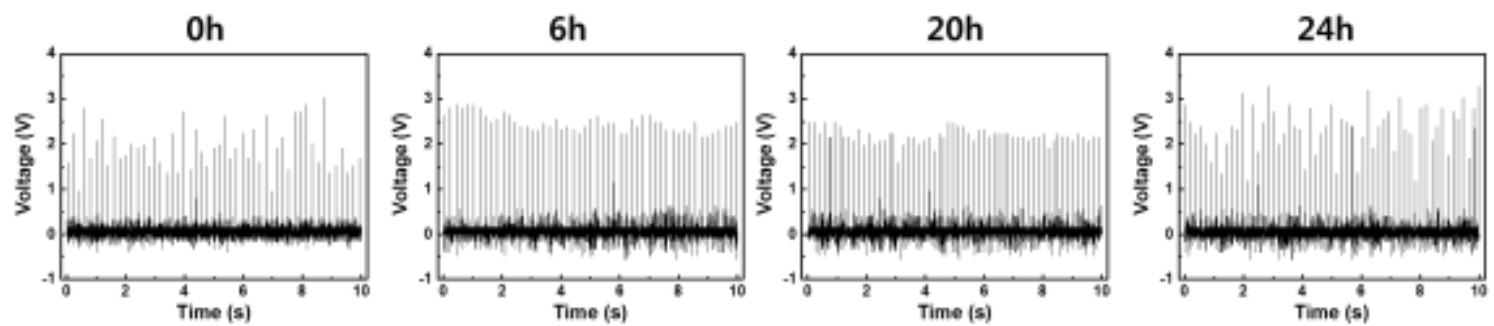

Figure S7. The $V_{O C}$ output in $0,6,20,24$ hours of super-hydrophobic surface in the CWTENG. 


\section{Geometry}

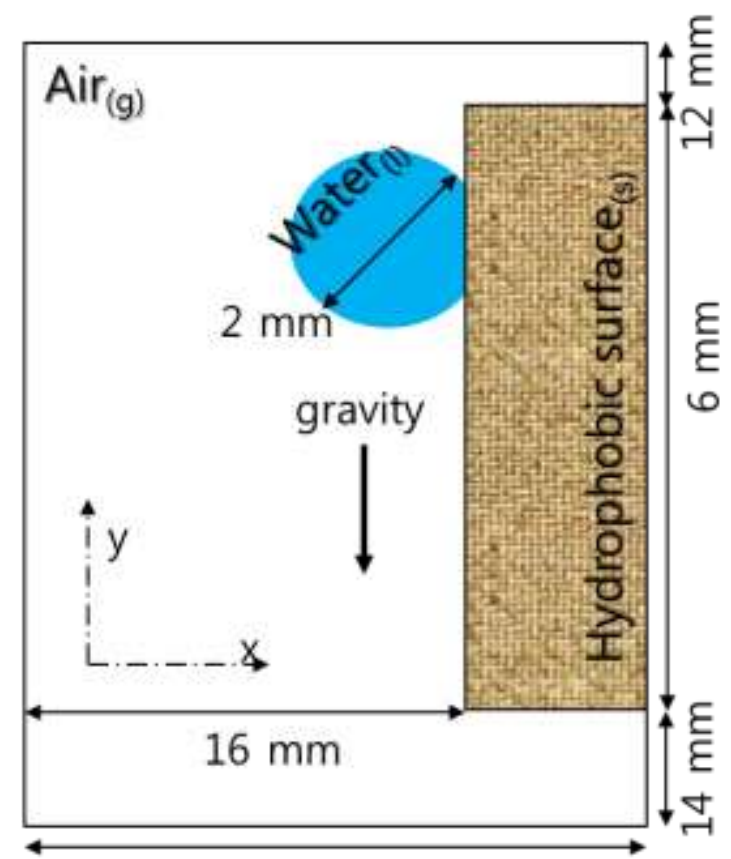

$18 \mathrm{~mm}$
Grid generation

Grid Type: Quadrilateral

Total Grid Number: 64,367

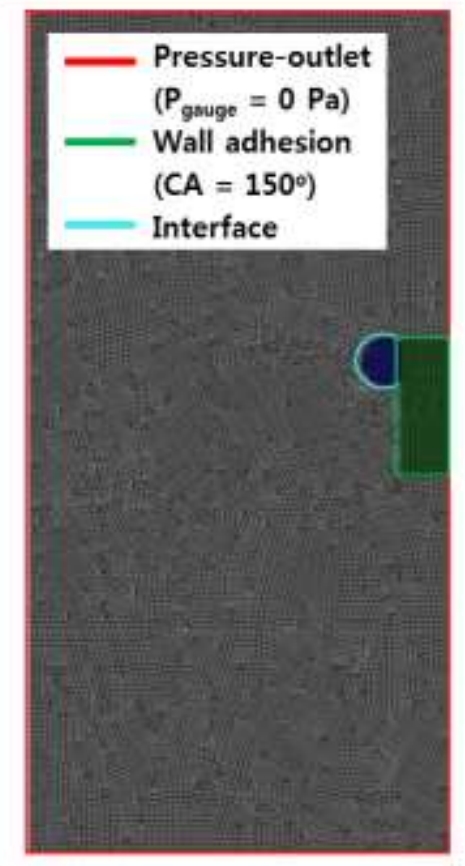

Figure S8. Pre-process for hydrophobic surface simulation. 


\section{Geometry (schematic)}

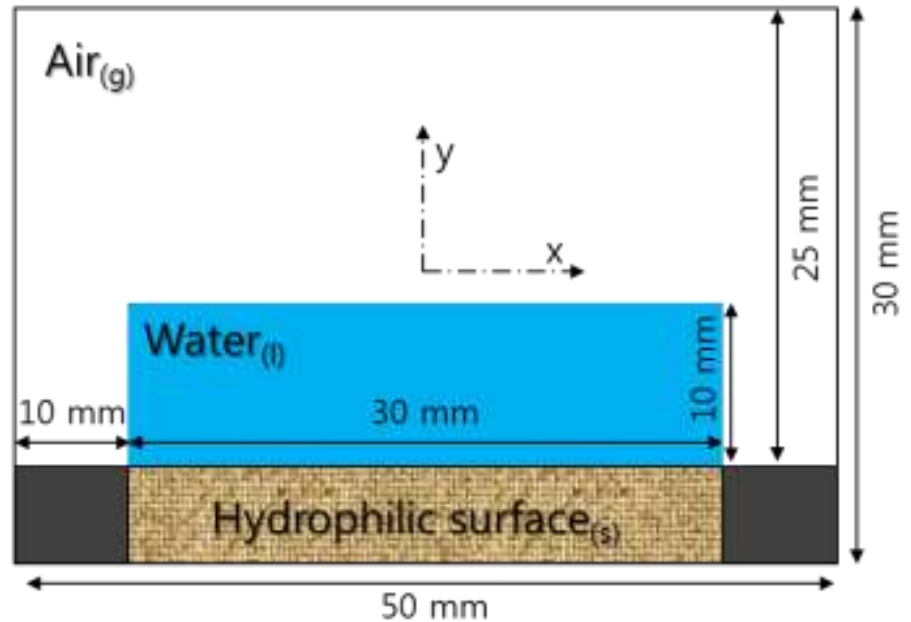

Grid generation

Grid Type: Quadrilateral Total Grid Number: 18,000

\section{Zone: Fluid $\mathrm{Al}_{2} \mathrm{O}_{3}$ Acryl}

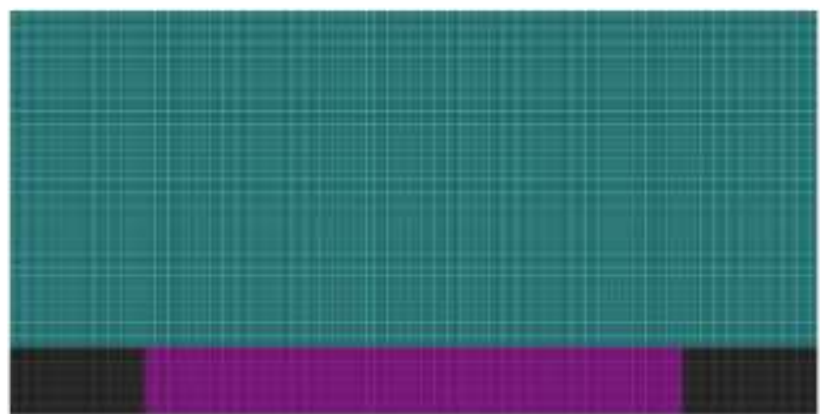

Figure S9. Pre-process for hydrophilic surface simulation. 


\section{Supporting Information - Simulation Methods}

To study the fluid flow in simulation, the governing equations of continuity, momentum, energy, and volume of fluid (VOF) model are employed in the FLUENT manual as follows[1]:

$\frac{\partial \rho}{\partial t}+\nabla \cdot(\rho \vec{u})=S_{m}$

$\frac{\partial}{\partial t}(\rho \vec{u})+\nabla \cdot(\rho \overrightarrow{u u})=-\nabla p+\nabla \cdot(\overline{\bar{\tau}})+\rho \vec{g}+\vec{f}_{s}$

where $S m$ is the mass source term; $\bar{\tau}$ is the stress tensor; $\rho \vec{g}$ and $\vec{f}_{s}$ are the gravity and the

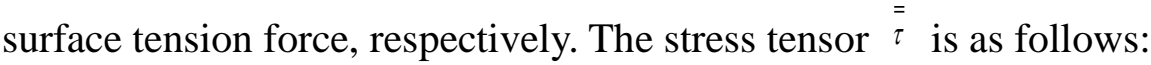

$\bar{\tau}=\mu\left[(\nabla \vec{u}+\nabla \vec{u})-\frac{2}{3} \nabla \vec{u} I\right]$,

where $I$ is the unit tensor.

The volume of fluid (VOF) method is adopted to model the multiphase flow, which traces the interface by finding the in-between values of the grids. This method solves the full Navier-Stokes equations for mass and momentum conservation by the advection of a scalar function, called a volume fraction $(\alpha)$. The volume fraction is defined as the percentage of volume covered by the multiphase within a computational cell with the total volume of the cell. The volume fraction advection equations are solved additionally by the following equations:

$$
\frac{\partial \alpha}{\partial t}+\nabla(\vec{u} \alpha) \oplus
$$


In the VOF method, one momentum equation is solved for gas and water, and the fluid properties are calculated according to the $\alpha$ value of the cell:

$\mu=\alpha \mu_{\text {water }}+(1-\alpha) \mu_{\text {air }}$

$\rho=\alpha \rho_{\text {water }}+(1-\alpha) \rho_{\text {air }}$.

The surface tension force, $\vec{f}_{s}$, in the momentum conservation equation can be expressed as follows:

$\vec{f}_{s}=2 \sigma \frac{\rho \kappa \nabla \alpha}{\rho_{\text {water }}+\rho_{\text {air }}}$

where $\kappa$ is the free surface curvature and is approximated as the divergence of the unit surface normal vector $\hat{n}$ :

$$
\kappa=\nabla \cdot \hat{n}
$$

The unit surface normal vector can be achieved from the prescribed value of the contact angle $\theta$ between the liquid and solid surface:

$\hat{n}=\frac{\nabla \alpha}{|\nabla \alpha|}=\hat{n}_{w} \cos \theta+\hat{n}_{t} \sin \theta$

where subscripts $w$ and $t$ are the wall and tangential direction, respectively.

The two-dimensional grid system was generated, and the total number of quadrilateral cells was 180,000. We used a commercial CFD software (Fluent V.16.2) to investigate the multiphase flow for an electrical potential generation. For the boundary conditions, the velocity of water is estimated as $5.233 \mathrm{~m} / \mathrm{s}$ which is the tangential velocity of rotating part having 400rpm. The initial height of the deposited water is assumed as $10 \mathrm{~mm}$. A time step is 
taken at $10^{-6} \mathrm{~s}$, considering the Courant number. The density of the water and the air is fixed as $998.2 \mathrm{~kg} / \mathrm{m}^{3}$ and $1.225 \mathrm{~kg} / \mathrm{m}^{3}$. The viscosity of the water and the air is also set as 0.001 $\mathrm{kg} / \mathrm{m} \cdot \mathrm{s}$ and $1.789 \times 10^{-5} \mathrm{~kg} / \mathrm{m} \cdot \mathrm{s}$. The surface tension at air-water interface is $0.0735 \mathrm{~N} / \mathrm{m}$. The contact angles of the hydrophilic and hydrophobic surface are fixed as $30^{\circ}$ and $150^{\circ}$, respectively, based on the experimental measurement. The SIMPLE scheme is employed for pressure-velocity coupling, and the first-order implicit is used for the transient formulation. The user-defined scalar (UDS) as an option in Fluent is embedded to solve the Gauss's law and Maxwell-Faraday equation for the generation of electrical potential. The computational domains and specific boundary conditions are described as shown in Supplementary Figure S8, 9. 